\title{
Self-Care Practice of Patients with Mechanical Heart Valve Prosthesis Accompanied in Nursing Consultation
}

\author{
Lidia Stella Teixeira de Meneses ${ }^{1}$, Francisca Elisangela Teixeira Lima ${ }^{2}$, \\ Sherida Karanini Paz de Oliveira1, Francisca Jane Gomes Oliveira1, \\ Maira di Ciero Miranda Vieira², Paulo Cesar Almeida3, \\ Viviane Martins da Silva ${ }^{2}$ \\ ${ }^{1}$ Federal University of Ceará, Fortaleza, Brazil \\ ${ }^{2}$ Department of Nursing, Federal University of Ceará, Fortaleza, Brazil \\ ${ }^{3}$ State University of Ceará, Fortaleza, Brazil \\ Email: lidiastellatm@yahoo.com.br, felisangela@yahoo.com.br, karanini@yahoo.com.br, \\ jane3876@hotmail.com, mairadi@bol.com.br, almeida@gmail.com, vivi@ufc.br
}

Received 8 July 2015; accepted 26 October 2015; published 29 October 2015

Copyright (C 2015 by authors and Scientific Research Publishing Inc.

This work is licensed under the Creative Commons Attribution International License (CC BY). http://creativecommons.org/licenses/by/4.0/

(c) (i) Open Access

\section{Abstract}

The objective was to evaluate the practice of self-care of patients with mechanical heart valve prosthesis. A descriptive, cross-sectional study developed in two outpatient valvopathies of teaching hospitals of Fortaleza/CE, from October 2013 to January 2014. The sample consisted of 127 patients with mechanical heart valve prosthesis. It was held an interview using instrument based on the Theory of Orem's Self-Care and Brazilian Guidelines for Valvular Heart Diseases. The data were presented in tables and charts. Results: Universal self-care practices of larger adhesion: body hygiene (97.6\% washed-haired, daily-bath $92.1 \%)$; oral hygiene (brushing teeth before sleeping, 87.4\%); fluid intake (drinking-water 95.2\%); food intake (salt intake $\leq 2 \mathrm{~g} / \mathrm{day}, 92.1 \%$, fruit and vegetable consumption 79.5\%); intestinal eliminations (without blood or mucus-96\%, non-parasitic 94.4\%); urinary elimination (no blood nor pus-96.8\%, urination 4 to 6 times a day, 96\%). As self-care developmental requirements predominated: never used illegal drugs, tobacco, alcohol, or stopped at the discovery of the disease (70\%). As self-care health deviation requirements we have: making use of certain medication at the right dose (95.2\%); attending medical appointments (cardiologist-92.1\%; nursing-84.2\%); INR control (identifies signs of bleeding-

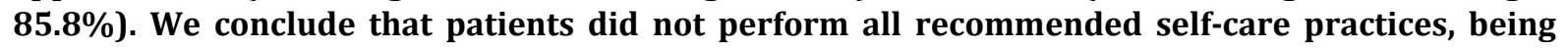
necessary to establish strategies to reduce the self-care deficit. 
Keywords

Nursing, Self-Care, Heart Valve

\section{Introduction}

For patient care with heart valve prosthesis, the nursing consultation comes as technological strategy of important and resolute care, backed by law, privative of the nurses, and offers numerous advantages in the care provided, facilitating health promotion, diagnosis and early treatment, in addition to prevention of avoidable situations [1].

In the year 2012, there were held in Brazil 7461 procedures for exchanging heart valve, with a predominance of procedures performed in the South, followed by the Northeast, where mortality rate remained at $8 \%$. This value is reduced when only analyzed the State of Ceara, which had a mortality rate of $4.62 \%$ over the same period [2].

For reduction of the mortality rate by heart valve diseases, it is critical that patients undergoing valve replacement surgery are provided with an accompaniment of a multidisciplinary team during the hospitalization period, continuing after discharge. The carefully planned, systematic, competent and accountable offered to the patient in all operative phases and appropriate outpatient follow-through education for health contributes to better and faster rehabilitation [3].

Given the above, it is necessary that the nurse, as a member of the multidisciplinary team, performs nursing consultation including educational, management, coordination and implementation of nursing care actions to encourage the patient, the family and the community to practice self-care [4]. The purpose of patient education is to increase his competence and confidence for self-management, in order to increase his responsibility and independence for self-care [5].

The self-care practice is essential to prevent complications and to maintain the clinical stability of the patient. So it is important that the health care team recognizes these practices in order to implement an effective and quality care for promotion of self-care and prevention of complications.

It is believed that the results of this study can contribute to the recognition of the patient's peculiarities with mechanical heart valve, as the evolution of these patients is directly related to the practice of self-care and adherence throughout their lives, requiring constant, rigorous monitoring of the multidisciplinary team; in addition to direct care to an individual and a holistic manner, focusing on the real needs of the patient.

The context described above gives rise to the following driver questioning of this research: Which are the self-care practices performed by patients with mechanical valve prosthesis, considering the universal requirements, developmental and health deviation? The objective was to evaluate self-care practices performed by patients with mechanical valve prostheses followed in nursing consultation.

\section{Methodology}

It is a descriptive study with cross-sectional design, quantitative, which took as a reference the theoretical model of Dorothea Orem [6], who determines as self-care concept the ability that the individual has to play or practice activities for his own benefit, in order to maintain life, health and their well-being.

The study was conducted in two anticoagulation clinics for patients with mechanical heart valve prosthesis located in the city of Fortaleza/CE, from October 2013 to January 2014. The population was composed of patients undergoing cardiac surgery mechanical valve replacement followed in these clinics.

The sample consisted of 127 patients who met the inclusion criteria: having performed heart surgery mechanical valve replacement; age $\geq 18$ years old; and attending the nursing consultation scheduled at the clinic of the institution in the period of data collection. And as exclusion criteria: to present on the day of consultation, hemodynamic instability. An explanation of the study by reading the consent form was performed. After signing the term began to collect data.

Patients were selected randomly, according to the schedule to the clinic in the period of data collection.

There was performed a structured interview with the use of a check-list type instrument, built based on the Theory of Orem's Self-Care [6] and the Brazilian Guidelines for Valvular Heart Diseases [7], divided into four 
parts, namely: socio-demographic data (gender, age, education, marital status, origin, family income, race, work activities, religious practice), clinical data (family history of cardiovascular disease, associated diseases, valvular heart disease, valve type, duration of surgery, medications in use and evaluation of the INR), self-care practices (universal, developmental and health deviation) and clinical consequents related to the deficit of self-care (bleeding events, thromboembolic, endocarditis, prosthetic dysfunction and prosthesis-patient mismatch). The instrument has not been validated.

Self-care practices include the universal self-care requirements, developmental and health deviations. Universal self-care requirements contemplated seven practices: body care, oral hygiene, fluid intake, food intake, intestinal and urinary elimination, physical exercise and sleep and rest. Self-care developmental requirement addressed a practice that included smoking, drinking and illicit drug use. The requirements related to health deviations contemplated four practices: regular use of medication, attendance at health team consultations, INR control, knowledge about the disease and treatment. In total, there were 12 self-care practices; each contained the essential actions of each practice, ranging from three to eight shares for self-care practice.

To evaluate self-care, each share corresponds to a point. However, the Brazilian Guidelines for Valvular Heart Diseases [7] state that the embolic and hemorrhagic events and endocarditis are the most common and clinically significant complications of long-term replacement valve. Furthermore, valvular heart disease is associated with traditional risk factors for atherosclerosis, such as hyperlipidemia, and smoking.

Thus, oral hygiene practices, food intake, smoking, alcohol consumption, illicit drugs, regular use of medication, INR control and attendance at health team consultations took different score, weighing 2. The score rangedfrom zero to 86 points.

After getting the data, according to the description of the procedures of self-care practices, the score achieved was categorized using the classification based on $\mathrm{P}_{33.3}$ and $\mathrm{P}_{66.6}$ tertiles. So the three bands were: poor to 58 points, regular between 59 and 68, and excellent 68 to 83 (which was the highest score found).

For statistical analysis there were calculated the averages and standard deviations of socio demographic variables. The tests Student $t$ and F of Snedecor (ANOVA) were used to comparing the means of quantitative variables. For the proportion of tests there were used the chi-square test and the likelihood ratio. There were considered as statistically significant the analysis with $p<0.05$.

The ethical and legal aspects were complied according to Resolution 466/2012 [8], being initiated the study after consideration and approval by the Ethics Committee of the Federal University of Ceara, under the Protocol $\mathbf{N}^{\circ} 422.098$.

\section{Results}

The predominant sociodemographic characteristics of patients with mechanical heart valve prostheses were women (56.7\%), the predominant age group was 40 - 59 years old $(50.4 \%)$, more than 5 years of education (69.2\%), married (63.8\%), family income of a minimum wage (53.5\%), non-white (55.1\%), absence of labor activity (80.3\%), Catholics (74\%).

For clinical data related to valve surgery there were more frequent due to valve disease mitral stenosis (12.6\%) and aortic insufficiency (12.6\%), the affected valve was the mitral (52\%), rheumatic fever history (56.6\%) and surgery was performed more than 10 years ago (40.5\%).

There were identified the practices of AC considering the requirements of universal self-care, developmental and health deviation of patients with mechanical valve prosthesis, according to the steps of the Theory of Orem's Self-Care [6], described in Tables 1-3.

Regarding the practice of personal hygiene, most patients performed the actions related to this practice. Among these actions, washed hair (97.6\%) and daily bath (92.1\%) were the most frequently performed.

In practice oral hygiene, it was found that $87.4 \%$ brushed their teeth before bed and $72.4 \%$ brushed their teeth after the main meals. But there was an AC deficit in relation to flossing, whose action was not performed by $70.9 \%$ of patients, as well as a high percentage of patients (71.7\%) still used toothpick after meals, which confers a risk for bleeding and endocarditis.

In relation to food ingestion, it was observed that the actions related to such practice were followed by most patients. However, there was an AC deficit especially in items: balanced intake of foods containing vitamin $\mathrm{K}$ (32.1\%) and it is approximately 5 to 6 meals per day (36.3\%).

For the practice of smoking, drinking and illegal drugs, it was found that most patients adhered to the neces- 
Table 1. Distribution of patients with valvular prosthesis, considering the requirements of universal self-care. Fortaleza/CE, Brazil, 2014.

\begin{tabular}{|c|c|c|}
\hline REQUIREMENTS OF UNIVERSAL SELF-CARE & $\mathbf{N}^{\circ}$ & $\%$ \\
\hline \multicolumn{3}{|l|}{ BODY HYGIENE } \\
\hline Hairwashed & 124 & 97.6 \\
\hline Daily bath & 117 & 92.1 \\
\hline Hands washed with soap and water as needed & 108 & 85 \\
\hline Nail clippings and clean (do not remove cuticles) & 73 & 57.4 \\
\hline Does shaving with electric appliance (avoids injury) & 29 & 22.8 \\
\hline \multicolumn{3}{|l|}{ ORAL HYGIENE } \\
\hline Brushesteethbeforesleeping & 111 & 87.4 \\
\hline Brushes teeth after the main meals & 92 & 72.4 \\
\hline Goes to the dentist twice per year & 44 & 34.6 \\
\hline Flossonce a day & 37 & 29.1 \\
\hline Does not use toothpick & 36 & 28.3 \\
\hline \multicolumn{3}{|l|}{ FLUID INTAKE } \\
\hline Ingest drinking water (boiled, filtered or mineral) & 121 & 95.2 \\
\hline Ingests water/fluids many times daily & 108 & 85 \\
\hline 8 to 10 glasses of water a day (2000 to $3000 \mathrm{ml}$ ), unless medical restriction & 97 & 76.3 \\
\hline \multicolumn{3}{|l|}{ FOOD INTAKE } \\
\hline Salt intake $\leq 2 \mathrm{~g} /$ day or abstention in food preparation & 117 & 92.1 \\
\hline Vegetables and fruits consumption daily & 101 & 79.5 \\
\hline Does not consume coffee or reduced consumption & 101 & 79.5 \\
\hline Predominant consumption of white meat & 100 & 78.7 \\
\hline Preferential use of vegetable fat & 96 & 75.5 \\
\hline Consume red meat at most 2 times a week & 95 & 74.8 \\
\hline Balanced consumption of foods containing vitamin $\mathrm{k}$ & 85 & 66.9 \\
\hline Makes about 5 to 6 meals a day (breakfast, snack, lunch, snack, dinner and supper) & 81 & 63.7 \\
\hline \multicolumn{3}{|l|}{ ELIMINATIONS } \\
\hline \multicolumn{3}{|l|}{ BOWEL } \\
\hline Without blood, mucus and pus secretion & 122 & 96 \\
\hline Absence of worms & 120 & 94.4 \\
\hline Feces pasty, brownish color and typical odor & 118 & 92.9 \\
\hline Frequency of evacuations of 1 to 2 times a day & 107 & 84.2 \\
\hline \multicolumn{3}{|l|}{ URINARY } \\
\hline Absence of blood or pus & 123 & 96.8 \\
\hline Urinary frequency of 4 to 6 times a day & 122 & 96 \\
\hline
\end{tabular}




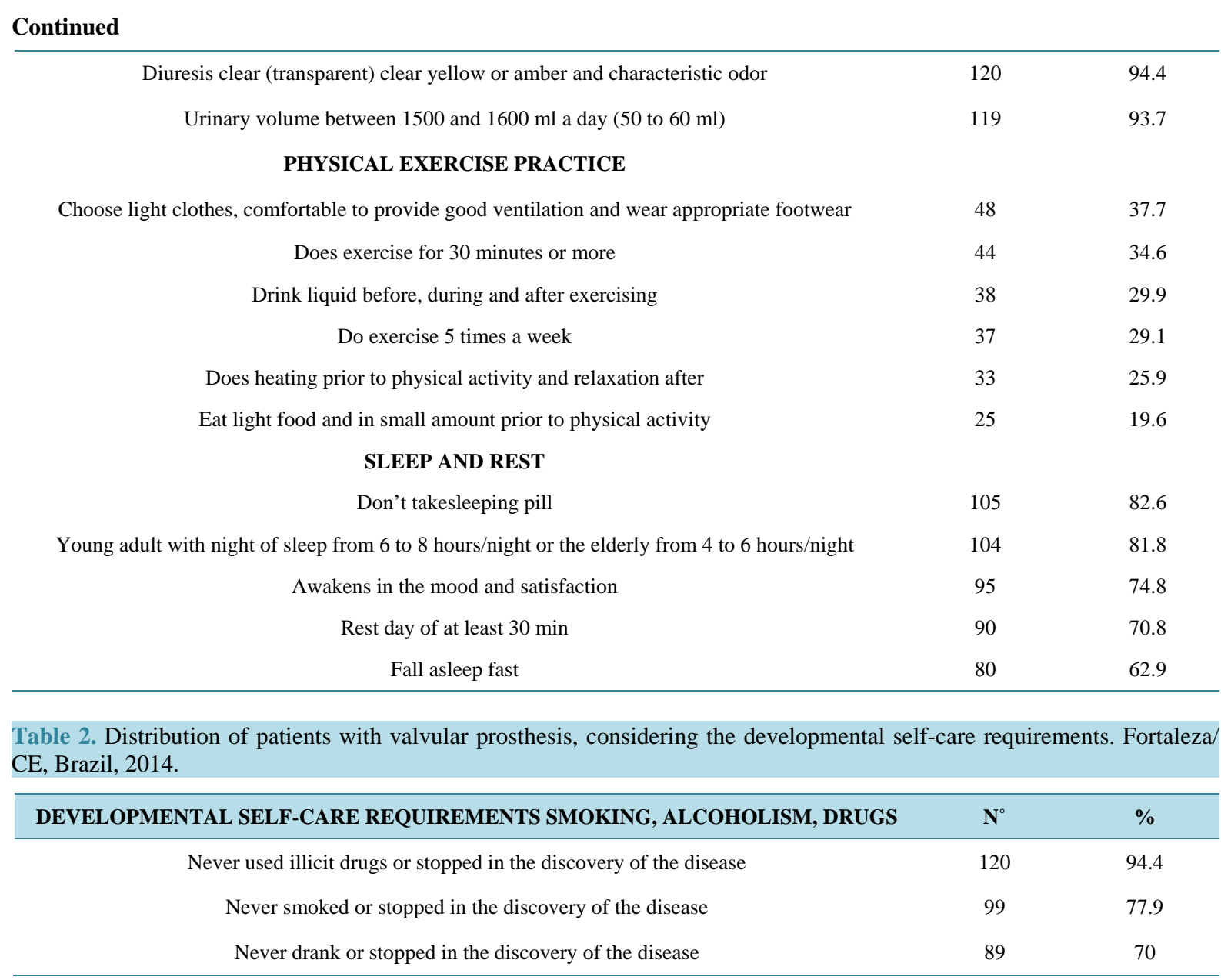

sary actions of this practice. All health professionals should conduct guidelines regarding the abstention of these drugs, since the practice is a risk factor for cardiovascular diseases.

For practical medication regularly, it was observed that most patients performed all related actions, such as certain medications (97.6\%) and right dose (95.2\%). However, there was an AC deficit in relation to the right time items and makes regular use of the medication, in which $24.5 \%$ and $22.9 \%$, respectively, reported not adhere to these actions.

Adherence to this practice is key to the recovery and prevention of complications in patients with mechanical heart prosthesis. Patients should be responsible for the use of medication and self-care that must be constantly encouraged by health professionals.

Regarding attendance at health team visits, it was found that most patients sought health care when presented any health change (92.9\%), and they attended the consultation with the cardiologist (92.1\%) and attended the consultation with the nurse (84.2\%).

Regarding the INR control, it was found that most identified signs of bleeding (85.8\%). However, there is a self-care deficit in actions related to laboratory control regular INR, to following a diet for patients using ACO and the caution with use of drugs that do drug interactions.

The low adherence to the actions related to the INR control may contribute to the increase in complications with the chronic use of ACO. Therefore, during the nursing consultation it is necessary to encourage these practices, with emphasis on drug interactions and adherence to diet for patients using ACO.

In Chart 1, the scores related to the practice of self-care are evident in patients with mechanical valve prosthesis, whose variation was 34 - 83 points. From the sum of the self-care practice of scores, it was found that $31.5 \%$ of patients underwent this practice scores excellent. However, $66.5 \%$ had self-care rated as fair or poor, which shows a self-care deficit in patients with mechanical heart valve prosthesis. 
Table 3. Distribution of patients with valvular prosthesis, considering the requirements of self-care health deviations. Fortaleza, CE/Brazil, 2014.

\begin{tabular}{|c|c|c|}
\hline HEALTH DEVIATION SELF-CARE REQUIREMENTS & $\mathbf{N}^{\circ}$ & $\%$ \\
\hline \multicolumn{3}{|l|}{ REGULAR USE OF MEDICATION } \\
\hline Makes use of the right medication & 124 & 97.6 \\
\hline Makes use of the right dose & 121 & 95.2 \\
\hline Makes regular use of medication prescribed & 98 & 77.1 \\
\hline Makes use on time & 96 & 75.5 \\
\hline \multicolumn{3}{|l|}{ ATTENDANCE AT HEALTH TEAM QUERIES } \\
\hline Searches for the doctor whenever needs & 118 & 92.9 \\
\hline Attends to the cardiologist consultation due systematically & 117 & 92.1 \\
\hline Attends to the nursing consultation due systematically & 107 & 84.2 \\
\hline Attends to the specialist's consultation due systematically, according to his needs & 69 & 54.3 \\
\hline \multicolumn{3}{|l|}{ CONTROL OF INR } \\
\hline Identifies signs of bleeding & 109 & 85.8 \\
\hline Be careful with the use of drugs that cause drug interaction with the ACO & 85 & 66.9 \\
\hline Follow the diet for patients using ACO oriented & 82 & 64.5 \\
\hline Performs laboratory control of INR regularly & 57 & 44.8 \\
\hline \multicolumn{3}{|l|}{ KNOWLEDGE } \\
\hline Seeks information about the disease and treatment & 73 & 57.4 \\
\hline $\begin{array}{l}\text { Seeks knowledge about self-care practices for the prevention of disease } \\
\text { and promotion of health of the patient with mechanical valve prosthesis }\end{array}$ & 73 & 57.4 \\
\hline Seeks information about complications on the chronic use of anticoagulant & 71 & 55.9 \\
\hline
\end{tabular}

Chart 1. Distribution of patients about the scores on the scale of the practice of self-care. Fortaleza/CE, 2014.

\begin{tabular}{ccc}
\hline Self-carepractice & $\mathbf{N}^{\circ}$ & $\%$ \\
\hline Excellent (69 - 83 points) & 40 & 31.5 \\
Regular (59 - 68 points) & 42 & 33.1 \\
Deficient (Até 58 points) & 45 & 35.4 \\
\hline
\end{tabular}

\section{Discussion}

The prevailing socio-demographic characteristics of patients with mechanical valve prostheses were female, aged between 40 and 59 years, more than five years of study, being married, coming from Fortaleza, household income of 1 minimum wage, not white, does not practice any labor activity, catholic.

With regard to practice personal hygiene, most patients adhered to the essential actions for this practice. Body hygiene is an important condition to prevent infection and to maintain a healthy life for patients.

Inadequate body hygiene was related to the use of handheld device for hair removal (blades). The use of these devices for this purpose should be discouraged and encouraged the use of electrical appliances or depilatory creams, whereas patients with chronic use of ACO at increased risk of bleeding that can occur with the use of the blade [7].

Oral hygiene was practiced by most patients. Maintaining a good hygiene and oral health aims to reduce risk of infective endocarditis, because of daily routine activities such as brushing and gargling (20\% to $68 \%$ ), tooth- 
pick use (20\% - 40\%) and even mastication (7\% to 51\%) can cause bacteremia [9].

Much of the population has poor oral and low access to dental treatment health, maintaining high incidence of streptococcal endocarditis in native valves and prostheses [7]. The low access to dental services was confirmed in this study, since $65.4 \%$ of patients did not perform regular visit to the dentist. This may be due to the difficulty of access to the service, as reported by the respondents.

Therefore, health services need to ensure better access of people to health information in health promotion context, and knowledge as a backdrop to the search for greater equity in oral health [10].

It was found that the practices related to food intake were followed by most patients, but an important practice that a deficit was balanced intake of vitamin K. This is alarming, since this excessive consumption can bring direct changes in values INR.

So it is up to the nurses during the follow-up visit to patients with mechanical valve prostheses, develop strategies for both patients and family members, encouraging them to adopt a balanced diet in order to minimize the risks of self-care related deficit this practice.

The practice of physical exercise was not carried out regularly by the participants. However, significant health benefits can be achieved with moderate physical activity every day of the week, such as 30 minutes of walking or 15 minutes of the race [11].

A low level of physical activity, defined by physical inactivity has been identified as a highly prevalent factor and, unfortunately, a risk factor for cardiovascular disease. Its relationship with hypertension, dyslipidemia, obesity and type 2 diabetes and, consequently, cardiovascular morbidity and mortality, physical inactivity should be treated as a public health problem [11].

With respect to smoking, it was observed that most patients reported complete abstention from this practice. However, it was found that there were still patients who were tobacco use. Data estimate that smoking is responsible for about 5.4 million deaths/year worldwide [12].

Thus, cigarette use should be discouraged by health professionals, being a completely avoidable practice and should be abolished people's lives, since there has been a reduction of 50\% of morbidity and mortality from cardiovascular disease after smoking cessation [13].

Regarding alcohol use, $30 \%$ of patients still reported use of alcohol, even after the discovery of the disease. This practice should be discouraged during the nursing consultation and monitored as increased intake of alcohol can cause liver dysfunction and, consequently, the ACO makes users more susceptible to coumarin expanding its share [14].

In addition, the abuse of alcohol is one of the triggering factors for coronary heart disease, since it favors the elevated blood pressure [15]. Therefore, we emphasize the need for health professionals carry out actions to investigate the use of alcohol in patients and encourage them to abstinence.

The illicit drugs were not used by most patients, but it was noted that $5.6 \%$ of the patients have not abstained from this practice. She should be discouraged, since drug addicts patients are more likely to develop acute infectious endocarditis [16].

The low adhesion to the use of prescription drugs and hence the proposed clinical treatment has a negative impact on all aspects of health care, cost overruns and under-utilization of available treatment resources, as well as serious consequences to patients and an increase in negative events in chronic diseases [17].

Attendance at health team consultations was held by most patients. But there was a self-care deficit in relation to attendance at nursing consultations in valvular heart disease clinic, to which $15.8 \%$ of patients did not attend regularly.

The nursing consultation is in addition to an aid action, a learning activity in which nurse and patient interact, seeking to solve the problems identified [18]. And for our patients, the nursing consultation is intended to guide the patient with respect to AC practices necessary to prevent complications and improve the quality of life.

A study that aimed to evaluate the effectiveness of therapy monitoring oral anticoagulant through valve disease clinics as a way of monitoring of therapy with oral anticoagulants compared to monitoring by the attending physician of the patient, found that there are obvious benefits in the effectiveness of monitoring through the follow-up valve disease clinics [19].

Thus, it is crucial that the implementation of valve disease clinics, since it is a privileged way of monitoring this type of therapy, since it is known that there is a clear relationship between an adequate anticoagulation control and a lower incidence of thrombotic or hemorrhagic complications [19].

Regarding the INR control, it was found that most knew identify signs of bleeding. However, there was a 
self-care deficit with respect to laboratory control of INR regularly, verifying that $55.2 \%$ of patients did not perform regularly the exam. It is recommended that the INR monitoring is carried out monthly, even if it remains in the ideal therapeutic range for long periods [20].

Other AC deficits were identified in this practice with respect to adherence to specific diet for patients using ACO and caution with the use of drugs that make interaction with the ACO.

These deficits should be monitored by health staff accompanying patients with mechanical valve prostheses. The causes of instability of oral anticoagulation therapy are multiple, mainly including interaction with other drugs and dietary changes or inconsistencies [21].

The importance of diet is often cited education programs for patients in outpatient anticoagulation therapy, and various nutrients, especially vitamin $\mathrm{K}$, they have been reminded in the interactions nutrients/drug with coumarin derivatives [22].

With respect to drugs that do interact with the ACO as antibiotics, anti-inflammatories, for example, these drugs can either potentiate, or inhibit the coagulation activity [14]. In this study, 33.1\% of patients had no caution when using any other medication, this fact related to low adherence to the diet for patients using ACO, which was observed in $35.5 \%$ of patients, directly affecting control INR.

\section{Conclusions}

The results of the study show that patients with mechanical valve prosthesis present self-care deficit. This result reinforces the importance of guidance and monitoring of a multidisciplinary team, in specialized clinics, based on implementation of educative actions on a regular basis to meet the needs and characteristics of each one, favoring the practice of self-care and improving the quality of life of patients and their families.

It is believed that the identification of self-care deficits can enable the development of a care plan targeted to the individual needs and specific to each, in order to minimize the existing deficiencies, as well as to motivate and guide individuals to carry out self-care.

In addition, this study will contribute to the construction of knowledge about the practice of self-care of patients with mechanical valve prosthesis and will guide the multidisciplinary team that accompanies these patients in specialized clinics.

The limitation of this research is on the aspect: the instrument has been made based according to Brasilian Guidelines for Valvular Heart diseases and is not yet an instrument validated, which reduces its reliability. So it is necessary to use a study to validate this instrument.

\section{References}

[1] Oliveira, S.K.P., Queiroz, A.P.O., Matos, D.P.M., Moura, A.F. and Lima, F.E.T. (2012) Temas abordados na consulta de enfermagem: Revisão integrativa da literatura. Revista Brasileira de Enfermagem, 65, 155-161.

[2] Ministério da Saúde (Brasil) (2013) Indicadores de Mortalidade: Taxa de mortalidade específica por doenças cerebrovasculares. Brasília: Ministério da Saúde_DATASUS; 2013. Acesso em 02 de março de 2013. Datasus. www.datasus.gov.br

[3] Carvalho, L.D.P., Mamede, M.V. and Araújo, M.R.O. (2011) Conhecimento de pacientes sobre o processo de autocuidado em pós—operatório de cirurgia cardíaca. Cadernos de Pesquisa, 18, São Luis; dez.Especial.

[4] Custódio, I.L. (2012) Avaliação do autocuidado de pacientes após transplante cardíaco acompanhado na consulta de enfermagem. 2012. 136f. Dissertação (Mestrado em enfermagem). Universidade Federal do Ceará, Fortaleza.

[5] Bastable, S.B. (2010) O enfermeiro como educador: princípios de ensino-Aprendizagem para a prática de enfermagem. 3rd Edition, Artmed, Porto Alegre.

[6] Orem, D.E. (1995) Nursing Concepts of Practice. 5th Edition, Mosby, Boston.

[7] Tarasoutchi, F., Montera, M.W., Grinberg, M., Barbosa, M.R., Piñeiro, D.J., Sánchez, C.R.M., Barbosa, M.M., Barbosa, G.V., et al. (2011) Diretriz Brasileira de Valvopatias-SBC 2011/I Diretriz Interamericana de ValvopatiasSIAC 2011. Arquivos Brasileiros de Cardiologia, 97, 1-67.

[8] Ministério da Saúde (Brasil) (2012) Conselho Nacional de Ética em Pesquisa—CONEP. Resolução No. 466/2012: Sobre pesquisa envolvendo seres humanos.

[9] Kiyose, A.T., Mitre, N. and Kasinski, N. (2009) Tratamento da Endocardite Infecciosa. In: Serrano Jr., C.V., Timerman, A. and Stefanini, E., Eds., Tratado de Cardiologia SOCESP, 2nd Edition, Manole, Barueri.

[10] Hainkal, D.S., Martins, A.M.E.B.L., Aguiar, P.H.S., Silveira, M.F., Paula, A.M.B. and Ferreira, E.F. (2014) O acesso à 
informação sobre higiene bucal e as perdas dentárias por cárie entre adultos. Ciência \& Saúde Coletiva, 19, 287-300.

[11] Matos, L.D.N.J., Trombeta, I.C. and Negrão, C.E. (2009) Sedentarismo e Beneficios da Atividade Física. In: Serrano, J.R., Timerman, A. and Stefanini, E., Eds., Tratado de Cardiologia SOCESP, 2nd Edition, Manole, Barueri, 517-526.

[12] World Health Organization (2008) Report on the Global Tobacco Epidemic, 2008: The MPOWER Package. Word Health Organization, Geneva.

[13] Wilson, K., Gibson, N., Willan, A. and Cook, D. (2000) Effect of Smoking Cessation on Mortality after Myocardial Infarction: Meta-Analysis of Cohort Studies. Archives of Internal Medicine Journal, 160, 939-944. http://dx.doi.org/10.1001/archinte.160.7.939

[14] Klack, K. and Carvalho, J.F. (2006) Vitamina K: Metabolismo, fontes e interação com o anticoagulante varfarina. Revista Brasileira de Reumatologia, 46, 398-406. http://dx.doi.org/10.1590/S0482-50042006000600007

[15] Sociedade Brasileira de Cardiologia/Sociedade Brasileira de Hipertensão/Sociedade Brasileira de Nefrologia (2010) VI Diretrizes Brasileiras de Hipertensão. Arquivos Brasileiros de Cardiologia, 95, 1-51.

[16] Tarasoutchi, F. and Grinberg, M. (2001) Emergências em valvopatias. In: Lage, S.G. and Ramires, J.A.F., Eds., Cardiologia no internato: Bases teórico-práticas, Editora Atheneu, São Paulo.

[17] Esmerio, F.G., Souza, E.M., Leiria, T.L., Lunelli, R. and Moraes, M.A. (2009) Uso crônico de anticoagulante oral: Implicações para o controle de níveis adequados. Arquivos Brasileiros de Cardiologia, 93. http://dx.doi.org/10.1590/S0066-782X2009001100017

[18] Vanzin, A.S. and Nery, M.E.S. (2000) Consulta de enfermagem: uma necessidade social? 2nd Edition, RM\&L, Porto Alegre.

[19] Barreira, R., Ribeiro, J., Farinha, M., Martins, R., Rodrigues, I., Mendes, Z. and Crespo, F. (2004) Monitorização da Terapêutica com Anticoagulantes orais: Consulta de anticoagulaçãovs Médico Assistente. Acta Médica Portuguesa, 17, 413-416.

[20] Meneghelo, Z.M., Liporace, I.L. and Barroso, C.M.Q. (2009) Terapia Antitrombótica nas doenças valvares. In: Serrano Jr., C.V., Timerman, A. and Stefanini, E., Eds., Tratado de Cardiologia SOCESP, 2nd Edition, Manole, Barueri, 13671388.

[21] Rohde, L.E., Assis, M.C. de and Rabelo, E.R. (2007) Dietary Vitamin K Intake and Anticoagulation in Elderly Patients. Current Opinion in Clinical Nutrition \& Metabolic Care, 10, 1-5. http://dx.doi.org/10.1097/mco.0b013e328011c46c

[22] Booth, S.L., Charnley, J.M., Sadowski, J.A., Saltzman, E., Bovill, E.G. and Cushman, M. (1997) Dietary Vitamin K1 and Stability of Oral Anticoagulation: Proposal of a Diet with Constant Vitamin K1 Content. Thrombosis and Haemostasis, 77, 504-509. 